\title{
Immunotherapy in Gastrointestinal Cancers
}

\author{
Melanie Güthle Thomas Ettrich Thomas Seufferlein
}

Internal Medicine I, University Hospital of UIm, Ulm, Germany

\section{Keywords}

Immunotherapy · Gastrointestinal cancer · MSI · PD-1 • CTLA-4

\begin{abstract}
Background: Gastrointestinal malignancies have both a high incidence rate and a high mortality rate. Immuno-oncological treatment approaches are becoming increasingly established in the treatment of gastrointestinal cancers. Summary: In this review, we give an overview of the types and effects of immunotherapies. We focus on recent studies on immunotherapies with special attention to immune checkpoint inhibition in carcinomas of the esophagus and stomach, the hepato-pancreatico-biliary system, and the colorectum including the anal channel in the metastatic setting, and we show their achievements but also their limitations. In an outlook, we discuss new approaches in immunotherapy like CAR T-cell therapy and oncolytic viruses. Key Messages: Gastrointestinal cancers show overall moderate response rates to immunotherapy. Nevertheless, subgroups such as DNA mismatch repair-deficient or microsatellite-instable tumors particularly benefit from the immune checkpoint blockade. Further studies are ongoing.

(c) 2020 S. Karger AG, Basel
\end{abstract}

\section{Introduction}

Immunotherapy has gained a significant value in the treatment of various solid tumors. Also for the treatment of some gastrointestinal cancers, the efficacy of immuno- therapy could be shown in recent years. Gastrointestinal malignancies have both a high incidence rate and a high mortality rate, and there is a definite need for novel therapeutic approaches. The majority of immunotherapies in gastrointestinal cancers are directed against immune checkpoints.

\section{Immune Surveillance}

Immunotherapy in cancer is based on the fact that tumors can be recognized as foreign and can be attacked by an activated immune system. Given the idea of the socalled immune surveillance, despite initially successful tumor elimination in the course of tumor development, increasing genetic instability of the malignant cell can lead to an equilibrium phase in which the tumor is immunologically controlled but not completely eliminated. The tumor escape phase follows the equilibrium phase, in which the immune system is no longer able to suppress the tumor. The immune escape succeeds in tumor cells through different mechanisms, either by reducing their own immunogenicity (downregulation of human leukocyte antigen 1) or actively suppressing the immune systems, for example by activating immune checkpoints [1].

\section{Cancer-Immunity Cycle}

Based on this concept, the immune response against a tumor is divided into several steps that have to be followed to enable immunological tumor elimination. In step 1, a tumor antigen is released from a dying tumor cell. This is recorded in step 2 by an antigen-presenting cell (dendritic cell), which migrates to a lymph node and presents the antigen to a $T$ cell in step 3 . This third step is 
Fig. 1. Overview of molecular targets and antagonistic antibodies in checkpoint inhibition. MHC, major histocompatibility complex; PD-L1, programmed cell death ligand 1; PD-1, programmed cell death; CTLA-4, cytotoxic T-lymphocyte-associated protein 4; ITIM, immunoreceptor tyrosine-based inhibitory motif; ITSM, immunoreceptor tyrosine-based switch motif; SHP-2, Src homology 2 (SH2) domain containing non-transmembrane PTP; P, phosphor.

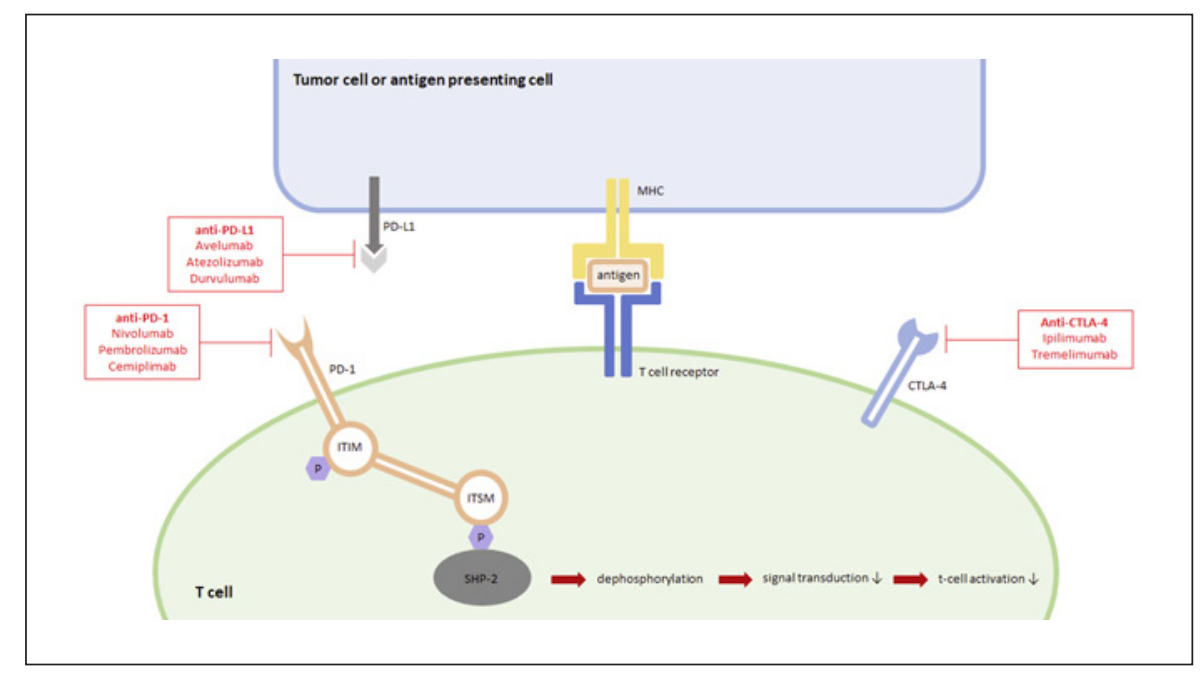

Table 1. T-cell receptors and agonistic and antagonistic antibodies

\begin{tabular}{ll}
\hline T-cell-activating receptors & Agonistic antibodies \\
\hline CD28 & \\
\hline OX40 & Not (yet) approved: \\
\hline GITR & - AMG228 \\
& - TRX518 \\
& - MK-4166 \\
& Not (yet) approved: \\
\hline CD137 & - Urelumab \\
\hline CD27 & \\
\hline HVEM & \\
\hline
\end{tabular}

essential because the ratio of effector CD8-positive T cells to regulatory $\mathrm{T}$ cells determines the further course of the immune reaction. When an antigen-presenting cell activates a naïve $\mathrm{T}$ cell using so called co-priming, this is called T-cell priming. Cytotoxic T-lymphocyte-associated protein 4 (CTLA-4-) antibodies (such as ipilimumab or tremelimumab) act primarily at this point. Subsequently, the activated $\mathrm{T}$ cell migrates to the tumor (step 4) and infiltrates it (step 5). In step 6, the tumor cell is recognized by the $\mathrm{T}$ cell using the $\mathrm{T}$-cell receptor. At this point, chimeric antigen receptor (CAR) T cells can attack. During this last step, the effector phase, the tumor cell is destroyed if the T cell is not deactivated by activating immune checkpoints. In the effector phase, programmed cell death (PD-1) receptor or ligand 1 (PD-L1)-blocking antibodies have their main effect. Following this step, the immunity cycle starts again with step 1 [2].

\section{Types of Immunotherapy}

Immunotherapy is a systemic treatment that either stimulates or suppresses the immune system to fight dis- eases such as cancer or infections, and it can be divided into passive and active forms. Approaches that already have intrinsic antineoplastic activity belong to the passive immunotherapies, for example adoptive T-cell transfer with CAR T cells or monoclonal antibodies such as the bi-specific T-cell engager such as blinatumomab. On the other hand, approaches that develop their effect through activation of the host immune system are active forms of immunotherapy. Examples of active immunotherapeutics are checkpoint inhibitors or tumor vaccines [3].

\section{Checkpoint Inhibitors}

The T-cell immune response is regulated among other factors by a complex interplay between activating and inhibiting coreceptors of the T cell [4] (Table 1). The two immune checkpoints CTLA- 4 and PD- 1 are of particular clinical importance. In the early phase of T-cell activation, the immune response can be suppressed by the interaction of CTLA- 4 with CD80 or CD86, which is expressed on antigen-presenting cells. This interaction is inhibited by antibodies such as ipilimumab and tremelimumab (Fig. 1).

In the effector phase of T-cell response, tumor cells can escape the immune response by the expression of PD-L1. With the help of the PD-1 receptor, T cells recognize PD-L1 and are thereby deactivated. A therapeutic intervention with radiation or chemotherapy can also lead to PD-L1 expression on tumor cells resulting in an immune escape. By blocking either PD-1 or PD-L1, a deactivation of the T cell is prevented, and this ideally leads to an immune response with destruction of the tumor cells (Fig. 1).

\section{Expression of $P D-L 1$}

PD-L1 expression is a potential biomarker for checkpoint inhibitors in clinical practice. However, this biomarker is suboptimal for several reasons, and frequently 
it cannot be used as a predictive biomarker: there are various and partly not validated antibodies available for the immunohistochemical detection of PD-L1. A cut-off value for PD-L1 positivity is not defined. PD-L1 can be detected on tumor cells, lymphocytes, and/or stroma cells. Furthermore, patients who do not exhibit PD-L1 expression in the tumor tissue may also benefit from treatment with PD-L1 inhibitors [5]. In addition, PD-L1 expression levels are dynamically regulated and modulated, e.g., by therapies. Thus, in many cases the immunohistochemical determination of PD-L1 expression does not really allow to select patients for checkpoint inhibitor treatment. PDL1 overexpression is an enrichment marker that defines a small patient population that is more likely to benefit from PD-(L)-1 blockade.

In 2017, the combined positive score (CPS) for the evaluation of PD-L1-expression in solid tumors by immunohistochemistry was developed as a more reproducible scoring method. CPS is evaluated based on the number of PD-L1-positive cells (tumor cells, lymphocytes, and macrophages) in relation to the total number of tumor cells. PD-L1 positivity is defined as CPS $\geq 1$, a high expression of PD-L1 as CPS $\geq 10$ [6].

\section{Tumor-Infiltrating Lymphocytes}

Infiltration of the tumor by lymphocytes is decisive for an adequate immune response. The absence of tumorinfiltrating lymphocytes indicates inadequate T-cell priming or immunosuppressive properties of the tumor microenvironment. It has been shown for various tumor entities that a high number of tumor-infiltrating lymphocytes correlates with prognosis and can predict the response to immunotherapy [7].

\section{Mutational Burden}

Tumors with a high mutational burden show in general a good response to checkpoint inhibitors. Conceptually, a high genomic heterogeneity leads to the expression of many neoantigens on the tumor cells, which are recognized as "foreign" by the immune system leading to a strong immune response against the tumor cells.

\section{Mismatch Repair Deficiency}

Deficiency of the mismatch repair system is closely linked to mutational burden. As a result, microsatellite instability (MSI) and, thus, a higher mutation rate occur. In microsatellite instable (MSI-H) or DNA mismatch repair-deficient (dMMR) tumors, there is a high rate of neoantigens and, thereby, a high rate of tumor infiltration by activated $\mathrm{T}$ cells $[8,9]$. A practice-changing study on colorectal cancer (CRC) patients showed that the overall tumor response rate (ORR) correlated with the MSI and dMMR status of the tumor. Patients with MSI-H CRC had an ORR in response to checkpoint inhibitor therapy of $40 \%$, whereas no response was observed in any patient who had a stable mismatch repair system [10]. Similar results were obtained in other tumors. Therefore, the US Food and Drug Administration (FDA) approved pembrolizumab for the treatment of tumors with dMMR as a first-line treatment independently of the tumor entity.

This article will focus on the status of immunotherapy in carcinomas of the esophagus and stomach, hepatopancreatico-biliary carcinomas, and CRC and anal cancer in the metastatic setting.

\section{Carcinomas of the Upper Gastrointestinal Tract}

\section{Immunotherapy in Esophagogastric Adenocarcinoma}

Four subtypes of esophagogastric cancers can be distinguished at the molecular level: tumors with chromosomal instability (50\%), MSI-H tumors (22\%), EpsteinBarr virus-positive (8\%), and genomically stable tumors (20\%) [11]. MSI phenotype and Epstein-Barr virus-positive gastric cancers are immunogenic subtypes with a high neoantigen burden and PD-L1 expression, making them eligible for treatment with anti-PD-1 immune checkpoint inhibitors [12]. Of note, only $5 \%$ of all metastatic and $<20 \%$ of all localized tumors are MSI-H and/or Epstein-Barr virus positive [13].

The KEYNOTE-059 trial, on previously untreated locally advanced or metastatic esophagogastric cancers, showed that treatment with pembrolizumab alone achieves an ORR of $26 \%$ with an overall survival (OS) rate of $63.6 \%$ after 1 year ( $40.1 \%$ after 2 years) in 31 patients with PD-L1-positive adenocarcinomas [14]. This approach was pursued in one of three study arms in the KEYNOTE-062 study. First results showed that monotherapy with pembrolizumab was not inferior compared to chemotherapy in terms of OS in PD-L1-positive tumors (CPS $\geq 1)$. In CPS $\geq 10$ tumors, pembrolizumab showed an improvement in OS compared to chemotherapy alone (17.4 vs. 10.8 months; HR 0.69) [15]. In the second-line setting, monotherapy with pembrolizumab was not inferior compared to chemotherapy in terms of OS in PD-L1-positive tumors (CPS $\geq 1$ ). In CPS $\geq 10$ tumors, the pembrolizumab group showed an OS of 17.4 months versus 10.8 months in the chemotherapy group [16].

The effect of nivolumab monotherapy in esophagogastric cancer refractory to or intolerant of at least two previous chemotherapy regimens is comparable to that of pembrolizumab, but the impact of $\mathrm{PD}-\mathrm{L} 1$ expression on efficacy is contradictory. In the ATTRACTION-2 study, nivolumab monotherapy increased the 12-month OS in an Asian collective to 27 versus $11 \%$ with placebo (HR $0.63, p<0.0001$ ), independently of the PD-L1 status, Lauren classification, and localization of the tumor [17]. In 
the CheckMate-032 study, the ORR was higher in PD-L1positive tumors (27 vs. 12\%) in Western patients [18]. In the KEYNOTE-059 trial, pembrolizumab in the thirdline setting (or later) in non-Asian patients showed response rates of $12 \%$ with a median OS of 5.6 months [19].

However, some trials showed negative results. In the KEYNOTE-061 trial (with pembrolizumab versus placebo, second line in 592 patients), the primary endpoint of improved OS and progression-free survival (PFS) was not met by the whole study group. In patients whose tumors overexpressed PD-L1 (CPS $\geq 10$ ), OS was significantly improved ( $n=53 / 296 ; 18 \%$; HR 0.64) [20]. The JAVELIN study showed no difference in OS between avelumab and chemotherapy with paclitaxel or irinotecan; however, in the PD-L1-positive subgroup, there was evidence for a predictive role of PD-L1 expression regarding OS [27].

In a small cohort $(n=25)$ of the KEYNOTE-059 study with PD-L1-positive and HER-2-negative tumors, the combination of pembrolizumab with chemotherapy (fluoropyrimidine and platinum) achieved an ORR of $60 \%$ in the whole population $(73.3 \%$ in the PD-L1-positive tumors vs. $37.5 \%$ in the PD-L1-negative tumors) [18]. OS or PFS in the pembrolizumab plus chemotherapy arm were not improved compared to chemotherapy alone [28].

Based on the results of the KEYNOTE-059 study, the anti-PD-1 monoclonal antibody pembrolizumab was approved by the FDA in 2017 for the treatment of refractory advanced gastric adenocarcinomas expressing PDL1 (as determined by an FDA-approved companion diagnostic test) and refractory advanced solid tumors with dMMR/MSI-H [19, 21].

Immunotherapy in Esophageal Squamous Cell Cancer

There are promising results for the use of anti-PD-1/ anti-PD-L1 antibodies in squamous cell cancer of the esophagus. The KEYNOTE-181 trial, of pembrolizumab versus investigator's choice chemotherapy (paclitaxel, docetaxel, or irinotecan) in the second-line treatment for advanced or metastatic squamous cell carcinoma and adenocarcinoma of the esophagus or esophagogastric junction, showed that pembrolizumab was superior to chemotherapy regarding OS in the CPS $\geq 10$ group. The 12 -month OS rate was $43 \%$ in this group compared to $20 \%$ in the control group [22]. The FDA has approved pembrolizumab for the treatment of PD-L1 expression in locally advanced or metastatic squamous cell carcinoma of the esophagus with disease progression after $\geq 1$ prior line of treatment. In patients with unresectable advanced or recurrent esophageal cancer (refractory or intolerant to fluoropyrimidine plus platinum), the ATTRACTION-3 study showed a significant improvement in median OS with nivolumab (10.9 months) versus chemotherapy (8.4 months) (HR 0.77, $p=0.019)$ [23].

\section{Hepato-Pancreatico-Biliary Carcinomas}

\section{Immunotherapy in Hepatocellular Carcinoma}

Up to $90 \%$ of all hepatocellular carcinomas (HCCs) arise in chronically inflamed livers. Thus, immunomodulating therapies appear to be a promising approach. The CheckMate-040 trial examined immune checkpoint blockade with nivolumab in patients with advanced HCC and progression after sorafenib (or sorafenib intolerance) [24]. In the expansion cohort, the response rate was 20\% with a 9 -month survival rate of $74 \%$. Interestingly, OS was higher in sorafenib-naïve patients (9.8 vs. 7.3 months, respectively). Based on these data, the FDA approved nivolumab for the treatment of patients with HCC who had been previously treated with sorafenib. A recent press release by the manufacturer revealed that the trial did not meet its primary endpoint. The KEYNOTE-224 study examined pembrolizumab in patients with HCC either refractory or intolerant to sorafenib. The ORR was $17 \%$, stable disease was achieved in 44\% [25]. However, also in this case, a press release at the beginning of 2019 stated that this study did not meet its co-primary endpoints either, a statistically significant difference in median OS and PFS.

In phase Ib clinical trials, the combination of atezolizumab and the anti-VEGF mAb bevacizumab [26] as well as the combination of nivolumab and ipilimumab showed promising response rates [27] that were further validated in phase III studies (e.g., the IMbrave150 trial or the CheckMate-9DW trial). First results of the IMbrave150 phase III trial were released at the end of 2019. Patients with treatment-naïve $\mathrm{HCC}$ received either a combination of atezolizumab and bevacizumab $(n=336)$ or sorafenib $(n=165)$. With a median follow-up of 8.6 months, the median OS with the combination was not estimable compared to 13.2 months with sorafenib (HR $0.58, p=0.0006$ ). The median PFS with the combination was 6.8 months versus 4.5 months with sorafenib (HR $0.59, p<0.0001$ ). The deterioration of quality of life was delayed in the group receiving the combination treatment. The superiority of atezolizumab and bevacizumab was particularly evident in a subgroup of virus-induced HCCs [28].

A phase II study investigating the combination of the anti-PD-L1 antibody durvulumab and the anti-CTLA-4 antibody tremelimumab for advanced-stage HCC (HIMALAYA) is ongoing.

\section{Immunotherapy in Pancreatic Cancer}

Pancreatic ductal adenocarcinoma (PDAC) is characterized by a low immunogenicity. Only $1 \%$ of the tumors are dMMR/MSI-H [29]. There is little evidence for using check point inhibition even in MSI-H PDAC. In a phase II basket trial, eight patients with dMMR PDAC achieved an objective response rate of $62 \%$ [21]. Two phase I trials 
with CTLA-4 inhibitors (ipilimumab or tremelimumab) in combination with gemcitabine showed no improvement of the ORR compared to gemcitabine alone [30,31]. In locally advanced or metastatic pancreatic adenocarcinoma, nivolumab and nab-paclitaxel \pm gemcitabine demonstrated disease control in 12 out of 17 patients in a phase I trial [32]. A recently published trial (KEYNOTE-158) in patients with advanced or metastatic noncolorectal MSI-H/dMMR carcinomas (27 different types of tumors) and disease progression or intolerance to prior standard therapy showed a benefit of therapy with the PD-1 antibody pembrolizumab in the whole patient group. However, in a subgroup of patients with MSI-H PDAC $(n=22)$, the median OS was 4 months with a median PFS of only 2.3 months. Thus, despite their MSI-H status, these patients do not seem to have a major benefit from checkpoint inhibitors [33].

\section{Immunotherapy in Biliary Tract Cancer}

So far, checkpoint inhibitors have shown modest activity in biliary tract cancer. In the KEYNOTE-028 study, 37 patients with PD-L1-positive biliary tract cancer after at least 1 prior therapy were treated with pembrolizumab. ORR was $17.4 \%$ [34]. In another study, nivolumab treatment showed an ORR of $20 \%$ in all patients with metastatic biliary tract cancer, independently of the PD-L1 status of the tumor [35]. In dMMR biliary tract cancers, pembrolizumab achieved an ORR of $53 \%$ in a phase II study [1]. These data are interesting, but only $3 \%$ of all biliary tract cancers exhibit a dMMR status [1].

\section{Colorectal and Anal Cancer}

\section{Immunotherapy in $C R C$}

About $95 \%$ of all CRC in the metastatic setting are microsatellite-stable (MSS)/mismatch repair-proficient (pMMR) tumors [21,36]. The Check-Mate-142 trial (phase II) evaluated single-agent nivolumab and nivolumab plus ipilimumab in patients with metastatic (m)CRC with either MSI-H/dMMR or MSS/pMMR status. Nivolumab monotherapy for 20 weeks showed a PFS rate in the dMMR group of $78 \%$ compared to $11 \%$ in the pMMR group. The therapy was well tolerated. Adverse events of all grades were fatigue (23\%), diarrhea (21\%), and rash (11\%). The most common grade 3 or 4 adverse event was an increase in lipase (8\%) [37]. There was evidence for a good efficacy of the combination of nivolumab with the CTLA-4 inhibitor ipilimumab from other cohorts of the Check-Mate-142 trial. Investigator-assessed ORR was 55\%, disease control rate for $\geq 12$ weeks was $80 \%$, and PFS rates were 76 and $71 \%$ ( 9 and 12 months, respectively) [38].

KEYNOTE-164 examined pembrolizumab monotherapy in heavily pretreated patients with MSI-H mCRC, achieving an ORR of $28 \%$ and an excellent 1 -year OS rate of $72 \%$ [10]. In contrast, MSS/pMMR tumors did not show any response to immune checkpoint inhibitors [36, 39].

To enhance the immunogenicity in MSS/pMMR mCRC, different combination trials were conducted. The combination of atezolizumab and the MEK inhibitor cobimetinib failed to improve outcome of patients with advanced mCRC compared to regorafenib [40]. A combination of FOLFOX (5-FU, folinic acid, and oxaliplatin) and PD-1/PD-L1 inhibitors ( \pm bevacizumab as inhibitor of vascular endothelial growth factor) showed an ORR of up to $50 \%$ and increased PFS (from 14 to 16 months) [41, 42]. Preliminary data from the AVETUX trial in patients with treatment-naïve MSS mCRC show an ORR of 75\% using a combination of FOLFOX, cetuximab, and the PDL1 antibody avelumab [43]. In contrast, the MODUL study showed no improvement of PFS by combining atezolizumab (anti-PD-L1) with maintenance therapy with fluoropyrimidines and bevacizumab after induction treatment with FOLFOX and bevacizumab as first-line treatment of mCRC [44].

In conclusion, all mCRCs should be tested for their microsatellite status. In MSI-H/dMMR CRC, checkpoint inhibitors should be used after therapy with fluoropyrimidine, oxaliplatin, and irinotecan. Currently, the value of checkpoint inhibitors in MSS CRC is still unclear.

\section{Immunotherapy in Anal Cancer}

The efficacy of pembrolizumab and nivolumab was shown in two single-arm trials for the treatment of refractory anal cancer. A multi-cohort phase Ib trial, in patients with PD-L1-positive squamous cell carcinoma of the anal canal (KEYNOTE-028), showed partial response with pembrolizumab in 4 out of 24 patients with advanced disease (RR 17\%), 10 patients (42\%) had stable disease. Median PFS was 3 months, and median OS was 9.3 months [45]. A phase II study of nivolumab, in patients with refractory metastatic squamous cell carcinoma of the anal canal, enrolled 37 patients unselected for PD-L1 status. There were 2 complete responses and 7 partial responses (RR 24\%). The median duration of response was 5.8 months among responders, the median PFS was 4.1 months, the median OS was 11.5 months [46]. Based on the data of these two trials, nivolumab and pembrolizumab were added to the National Comprehensive Cancer Network (NCCN) guidelines for systemic therapy of anal carcinoma in 2018.

\section{Conclusion}

A significant response to monotherapy with immune checkpoint inhibitors has only been observed in MSI-H gastrointestinal tumors so far. Microsatellite instability 
refers to a change in the length of short repetitive DNA sequences, the so-called microsatellites. The good response of MSI-H tumors is likely to be due to their high mutational burden, i.e., the number of mutations per mega base pair in the DNA of the tumor cell. Studies in recent years have shown that immunotherapies work much better against tumors that have many mutations, like malignant melanoma or tobacco-associated lung cancer. The determination of tumor mutational burden to identify subgroups of tumors, apart from MSI-H cancers that may benefit from immunotherapy, is not yet established in gastrointestinal malignancies.

Even among microsatellite-unstable tumors, there are tumors that do not respond well to immune checkpoint inhibitor treatment. For advanced CRC with the MSI-H phenotype, this was the case in up to half of all patients in previous studies. In PDAC checkpoint inhibition shows only rather moderate results even in MSI-H cancers. The challenge is to identify those patients with MSI-H tumors who are most likely to benefit from therapy with checkpoint inhibitors. So far, the only predictive biomarker in some gastrointestinal tumors is PD-L1. Additional biomarkers, also for checkpoint inhibitors that address other mechanisms than the PD1/PD-L1 system, are needed. These may come from genetic and epigenetic signatures as well as the analysis of the composition of the immune cells in the tumor and its microenvironment.

\section{New Approaches in Immunotherapy}

With the development of the technology for the production of CAR, a milestone was reached in the area of adoptive T-cell therapy. A CAR consists of a tumor-specific extracellular antibody fragment linked to an intracellular T-cell receptor fragment [47]. Autologous T cells must be removed from the patient and are genetically modified using a retroviral vector so that they express the desired CAR. The cells are then expanded ex vivo and reinfused into the patient. The advantages of this T-cell therapy are major histocompatibility complex-independent activation and a high tumor specificity; the disadvantages are the limitation to an extracellular antigen, high costs, and a high logistical effort. Spectacular success has been achieved with this form of adoptive cell therapy for hematological malignancies. Multiple early phase I studies demonstrate the efficacy of CAR T-cell therapy in preclinical models of solid tumors, and several randomized trials are ongoing.

Low immunogenicity may be overcome by vaccine immunotherapy aiming at immune response activation. The immunological mechanism of action of (genetically modified) oncolytic viruses is two-fold. First, these viruses replicate selectively in malignant cells and lead directly to tumor lysis. Second, as a result of virally induced cell lysis by the release of tumor-associated antigens, immunological alarm signals induce a systemic immune response [48]. Randomized trials on vaccination strategies in gastrointestinal malignancies are ongoing.

\section{Disclosure Statement}

The authors declare that there are no conflicts of interest regarding this article.

\section{Funding Sources}

Not applicable.

\section{Author Contributions}

M.G. and T.S. analyzed the literature and wrote the manuscript with input from T.E.

\section{References}

1 Ferris RL. Immunology and immunotherapy of head and neck cancer. J Clin Oncol. 2015 Oct;33(29):3293-304.

2 Chen DS, Mellman I. Oncology meets immunology: the cancer-immunity cycle. Immunity. 2013 Jul;39(1):1-10.

3 Galluzzi L, Vacchelli E, Bravo-San Pedro JM, Buqué A, Senovilla L, Baracco EE, et al. Classification of current anticancer immunotherapies. Oncotarget. 2014 Dec;5(24):12472-508.

4 Mellman I, Coukos G, Dranoff G. Cancer immunotherapy comes of age. Nature. $2011 \mathrm{Dec}$; 480(7378):480-9.

5 Brahmer J, Reckamp KL, Baas P, Crinò L, Eberhardt WE, Poddubskaya E, et al. Nivolumab versus Docetaxel in advanced squamous-cell non-small-cell lung cancer. N Engl J Med. 2015 Jul;373(2):123-35.

6 Kulangara K, Hanks DA, Waldroup S, Peltz L, Shah S, Roach C, et al. Development of the combined positive score (CPS) for the evaluation of PD-L1 in solid tumors with the immunohistochemistry assay PD-L1 IHC 22C3 pharmDx. J Clin Oncol. 2017;35(15_ suppl):e14589.

7 Tumeh PC, Harview CL, Yearley JH, Shintaku IP, Taylor EJ, Robert L, et al. PD-1 blockade induces responses by inhibiting adaptive immune resistance. Nature. 2014 Nov; 515(7528):568-71.

8 Tran E, Ahmadzadeh M, Lu YC, Gros A, Turcotte S, Robbins PF, et al. Immunogenicity of somatic mutations in human gastrointestinal cancers. Science. 2015 Dec;350(6266):1387-90.

9 Turajlic S, Litchfield K, Xu H, Rosenthal R, McGranahan N, Reading JL, et al. Insertionand-deletion-derived tumour-specific neoantigens and the immunogenic phenotype: a pan-cancer analysis. Lancet Oncol. 2017 Aug; 18(8):1009-21.
10 Diaz LA, Marabelle A, Delord JP, ShapiraFrommer R, Geva R, Peled N, et al. Pembrolizumab therapy for microsatellite instability high (MSI-H) colorectal cancer (CRC) and non-CRC. J Clin Oncol. 2017;35(15_ suppl):Abstract 3071.

11 Cancer Genome Atlas Research Network. Comprehensive molecular characterization of gastric adenocarcinoma. Nature. 2014 Sep; 513(7517):202-9.

12 Kim ST, Cristescu R, Bass AJ, Kim KM, Odegaard JI, Kim K, et al. Comprehensive molecular characterization of clinical responses to PD-1 inhibition in metastatic gastric cancer. Nat Med. 2018 Sep;24(9):1449-58.

13 Cristescu R, Lee J, Nebozhyn M, Kim KM, Ting JC, Wong SS, et al. Molecular analysis of gastric cancer identifies subtypes associated with distinct clinical outcomes. Nat Med. 2015 May;21(5):449-56. 
14 Wainberg ZA, Yoon HH, Catenacci DVT, Jalal SI, Muro K, Garrido M, et al. Efficacy and safety of pembrolizumab (pembro) alone or in combination with chemotherapy (chemo) in patients (pts) with advanced gastric or gastroesophageal (G/GEJ) cancer: long-term follow up from KEYNOTE-059. J Clin Oncol. 2019;37(15_suppl):Abstract 4009.

15 Tabernero J, Van Cutsem E, Bang YJ, Fuchs CS, Wyrwicz L, Lee KW, et al. Pembrolizumab with or without chemotherapy versus chemotherapy for advanced gastric or gastroesophageal junction (G/GEJ) adenocarcinoma: The phase III Keynote-062 study. J Clin Oncol. 2019;37(18_suppl):Abstract LBA4007.

16 Kojima T, Muro K, Francois E, Hsu CH, Moriwaki T, Kim SB, et al. Pembrolizumab versus chemotherapy as second-line therapy for advanced esophageal cancer: Phase III KEYNOTE-181 study. J Clin Oncol. 2019; 37(4_suppl):2.

17 Kang YK, Boku N, Satoh T, Ryu MH, Chao Y, Kato K, et al. Nivolumab in patients with advanced gastric or gastro-oesophageal junction cancer refractory to, or intolerant of, at least two previous chemotherapy regimens (ONO4538-12, ATTRACTION-2): a randomised, double-blind, placebo-controlled, phase 3 trial. Lancet. 2017 Dec;390(10111):2461-71.

18 Janjigian YY, Bendell J, Calvo E, Kim JW, Ascierto PA, Sharma P, et al. CheckMate-032 Study: Efficacy and Safety of Nivolumab and Nivolumab Plus Ipilimumab in Patients With Metastatic Esophagogastric Cancer. J Clin Oncol. 2018 Oct;36(28):2836-44.

19 Fuchs CS, Doi T, Jang RW, Muro K, Satoh T, Machado M, et al. Safety and Efficacy of Pembrolizumab Monotherapy in Patients With Previously Treated Advanced Gastric and Gastroesophageal Junction Cancer: Phase 2 Clinical KEYNOTE-059 Trial. JAMA Oncol. 2018 May;4(5):e180013.

20 Shitara K, Özgüroğlu M, Bang YJ, Di Bartolomeo $\mathrm{M}$, Mandalà M, Ryu MH, et al.; KEYNOTE-061 investigators. Pembrolizumab versus paclitaxel for previously treated, advanced gastric or gastro-oesophageal junction cancer (KEYNOTE-061): a randomised, open-label, controlled, phase 3 trial. Lancet. 2018 Jul;392(10142):123-33.

21 Le DT, Durham JN, Smith KN, Wang H, Bartlett BR, Aulakh LK, et al. Mismatch repair deficiency predicts response of solid tumors to $\mathrm{PD}$-1 blockade. Science. 2017 Jul;357(6349):409-13.

22 Shah MA, Adenis A, Enzinger, PC, Kojima R, Muro K, Bennouna J, et al. Pembrolizumab versus chemotherapy as second-line therapy for advanced esophageal cancer: Phase 3 KEYNOTE-181 study. J Clin Oncol. 2019; 37(15_suppl):4010.

23 Cho BC, Kato K, Takahashi M, Okada M, Lin CY, Chin K, et al. Nivolumab versus chemotherapy in advanced esophageal squamous cell carcinoma (ESCC): the phase 3 ATTRACTION-3 Study. Ann Onc. 2019; 30(suppl_5):v873-4.

24 El-Khoueiry AB, Sangro B, Yau T, Crocenzi TS, Kudo M, Hsu C, et al. Nivolumab in patients with advanced hepatocellular carcinoma (CheckMate 040): an open-label, non-comparative, phase $1 / 2$ dose escalation and expansion trial. Lancet. 2017 Jun;389(10088):2492-502.
25 Zhu AX, Finn RS, Edeline J, Cattan S, Ogasawara S, Palmer D, et al.; KEYNOTE-224 investigators. Pembrolizumab in patients with advanced hepatocellular carcinoma previously treated with sorafenib (KEYNOTE-224): a non-randomised, open-label phase 2 trial. Lancet Oncol. 2018 Jul;19(7):940-52.

26 Lee KH, Hsu CH, Lee MS, Ryoo BY, Verret W, $\mathrm{He} \mathrm{AR}$, et al. Atezolizumab + bevacizumab in hepatocellular carcinoma (HCC): safety and clinical activity results from a phase Ib study. Ann Oncol. 2018;29:29.

27 Yau T, Kang YK, Kim TY, El-Khoueiry AB, Santoro A, Sangro B, et al. Nivolumab (NIVO) + ipilimumab (IPI) combination therapy in patients (pts) with advanced hepatocellular carcinoma (aHCC): results from CheckMate 040. J Clin Oncol. 2019;37(15_suppl):4012.

28 Cheng AL, Qin S, Ikeda M, Galle P, Ducreux M, Zhu A, et al. LBA3 IMbrave150: efficacy and safety results from a ph III study evaluating atezolizumab (atezo) + bevacizumab (bev) vs sorafenib (Sor) as first treatment (tx) for patients (pts) with unresectable hepatocellular carcinoma (HCC). Ann Oncol. 2019; 30(suppl_9):ix186-7.

29 Humphris JL, Patch AM, Nones K, Bailey PJ, Johns AL, McKay S, et al.; Australian Pancreatic Cancer Genome Initiative. Hypermutation In Pancreatic Cancer. Gastroenterology. 2017 Jan;152(1):68-74.e2.

30 Wainberg ZA, Hochster HS, George B, Gutierrez $\mathrm{M}$, Johns ME, Chiorean EG, et al. Phase I study of nivolumab (nivo) + nab-paclitaxel (nab-P) \pm gemcitabine (Gem) in solid tumors: interim results from the pancreatic cancer (PC) cohorts. J Clin Oncol. 2017;35(4_suppl):412.

31 Marabelle A, Le DT, Ascierto PA, Di Giacomo AM, De Jesus-Acosta A, Delord JP, et al. Efficacy of Pembrolizumab in Patients With Noncolorectal High Microsatellite Instability/Mismatch Repair-Deficient Cancer: Results From the Phase II KEYNOTE-158 Study. J Clin Oncol. 2020 Jan;38(1):1-10.

32 Bang YJ, Doi T, Braud F, Piha-Paul S, Hollebecque A, Razak AR, et al. Safety and efficacy of pembrolizumab (MK-3475) in patients (pts) with advanced biliary tranct cancer: interim results of KEYNOTE-028. Eur J Cancer. 2015;51:S112.

33 Gou M, Zhang Y, Si H, Dai G. Efficacy and safety of nivolumab for metastatic biliary tract cancer. OncoTargets Ther. 2019 Jan;12:8617.

34 Le DT, Uram JN, Wang H, Bartlett BR, Kemberling $\mathrm{H}$, Eyring AD, et al. PD-1 Blockade in Tumors with Mismatch-Repair Deficiency. N Engl J Med. 2015 Jun;372(26):2509-20.

35 Gou M, Zhang Y, Si H, Dai G. Efficacy and safety of nivolumab for metastatic biliary tract cancer. OncoTargets Ther. 2019 Jan;12:861-7.

36 Le DT, Uram JN, Wang H, Bartlett BR, Kemberling $\mathrm{H}$, Eyring AD, et al. PD-1 Blockade in Tumors with Mismatch-Repair Deficiency. N Engl J Med. 2015 Jun;372(26):2509-20.

37 Overman MJ, McDermott R, Leach JL, Lonardi S, Lenz HJ, Morse MA, et al. Nivolumab in patients with metastatic DNA mismatch repair-deficient or microsatellite instabilityhigh colorectal cancer (CheckMate 142): an open-label, multicentre, phase 2 study. Lancet Oncol. 2017 Sep;18(9):1182-91.
38 Overman MJ, Lonardi S, Wong KY, Lenz HJ, Gelsomino F, Aglietta M, et al. Durable Clinical Benefit With Nivolumab Plus Ipilimumab in DNA Mismatch Repair-Deficient/Microsatellite Instability-High Metastatic Colorectal Cancer. J Clin Oncol. 2018 Mar;36(8):773-9.

39 Overman MJ, Kopetz ES, McDermott R, Leach J, Lonardi S, Lenz HJ, et al. Nivolumab \pm ipilimumab in treatment (tx) of patients (pts) with metastastic colorectal cancer (mCRC) with and without high microsatellite instability (MSI$\mathrm{H})$ : CheckMate-142 interim results. J Clin Oncol. 2016;34(suppl):Abstract 3501.

40 Eng C, Kim TW, Bendell J, Argilés G, Tebbutt NC, Di Bartolomeo M, et al.; IMblaze370 Investigators. Atezolizumab with or without cobimetinib versus regorafenib in previously treated metastatic colorectal cancer (IMblaze370): a multicentre, open-label, phase 3 , randomised, controlled trial. Lancet Oncol. 2019 Jun;20(6):849-61.

41 Bendell JC, Powderly JD, Lieu CH, Eckhardt SG, Hurwitz H, Hochster HS, et al. Safety and efficacy of MPDL3280A (anti-PDL1) in combination with bevacizumab (bev) and/or FOLFOX in patients (pts) with metastastic colorectal cancer (mCRC). J Clin Oncol. 2015; 33(3_suppl):704.

42 Sahdhda S, Noonan AN, Bekaii-Saab T, O'Neil BH, Sehdev A, Shaib WL, et al. A phase II study of pembrolizumab in combination with mFOLFOX6 for patients with advanced colorectal cancer. J Clin Oncol. 2017;35(15_ suppl):Abstract 3541 .

43 Stein A, Binder M, Al Batran SE, Hinke A, Waberer L, Goekkurt E, et al. Avelumab and cetuximab in combination with FOLFOX in patients with previously untreated metastatic colorectal cancer (mCRC): results of the safety run-in phase of the phase II AVETUX trial (AIO-KRK-0216). J Clin Oncol. 2018;36(15_ suppl):3561.

44 Grothey A, Tabernero J, Arnold D, De Gramont A, Ducreux MP, O'Dwyer PJ, et al. Fluoropyrimidine $(\mathrm{FP})+$ bevacizumab $(\mathrm{BEV})+$ atezolizumab vs FP/BEV in BRAFwt metastastic colorectal cancer (mCRC): Findings from Cohort 2 of MODUL - a multicentre, randomized trial of biomarker-driven maintenance treatment following first-line induction therapy. Ann Oncol. 2018;29(Suppl 8):viii714-5.

45 Ott PA, Piha-Paul SA, Munster P, Pishvaian $\mathrm{MJ}$, van Brummelen EM, Cohen RB, et al. Safety and antitumor activity of the anti-PD-1 antibody pembrolizumab in patients with recurrent carcinoma of the anal canal. Ann Oncol. 2017 May;28(5):1036-41.

46 Morris VK, Salem ME, Nimeiri H, Iqbal S, Singh P, Ciombor K, et al. Nivolumab for previously treated unresectable metastatic anal cancer (NCI9673): a multicentre, single-arm, phase 2 study. Lancet Oncol. 2017 Apr;18(4):446-53.

47 Bracci L, Schiavoni G, Sistigu A, Belardelli F. Immune-based mechanisms of cytotoxic chemotherapy: implications for the design of novel and rationale-based combined treatments against cancer. Cell Death Differ. 2014 Jan;21(1):15-25.

48 Kaufman HL, Kohlhapp FJ, Zloza A. Oncolytic viruses: a new class of immunotherapy drugs. Nat Rev Drug Discov. 2015 Sep;14(9): 642-62. 